\title{
Modeling of Heart Rate Variability and Respiratory Muscle Activity in Organophosphate Poisoned Patients
}

\author{
Maria Bernarda Salazar*, Mauricio Hernández, Miguel Ángel Mañanas and Cesar Cortés
}

\begin{abstract}
We propose an extended model of cardiovascular regulation to assess heart rate variability in patients poisoned with organophosphate during their treatment with mechanical ventilation. The model was modified to fit a population of twentyone (21) patients poisoned with organophosphorus compounds and undergoing mechanical ventilation. The extended model incorporated the respiratory muscle activity measured by surface electromyography for quantifying the vagal - sympathetic engagement during spontaneous breathing test. The order and structure of the parasympathetic and the sympathetic transfer function with respect to the original model were modified to a second - order system. In this extended model, the parameters related to the vagal - sympathetic response (corner frequency and constant gain) were correlated with respiratory muscle activity. When the diaphragm's contractions were stronger, the sympathetic corner frequency increased while the parasympathetic corner frequency and gain decreased. Thus the proposed model could be useful to improve the ventilatory support and pharmacological treatment for patients poisoned with organophosphorus compounds considering the vagal sympathetic response inferred from the respiratory muscle activity.
\end{abstract}

Index Terms - Heart rate variability, organophosphate poisoning, parasympathetic system, sympathetic system, surface electromyography, mechanical ventilation, spontaneous breathing test.

\section{INTRODUCTION}

$\mathrm{T}_{\mathrm{b}}^{\mathrm{h}}$ he cardiac activity and parasympathetic - sympathetic balance is assessed with the Heart Rate Variability (HRV) [1], measured during a time interval using non-invasive techniques. The cardiac contraction force and heartbeat speed are regulated by the Autonomic Nervous System (ANS), with mediation of the parasympathetic (PNS) and the sympathetic (SNS) nervous system. The periodic component of HRV ranges from 0.04 to $0.4 \mathrm{~Hz}$, where the high frequency (HF) range $(0.15$ to $0.4 \mathrm{~Hz}$ ) is mediated by the PNS [2], [3], and the low frequency (LF) range $(0.04$ to $0.15 \mathrm{~Hz})$ is generally mediated both by sympathetic and parasympathetic actions [2]. These systems usually have opposite effects on the body, the SNS

This study was partially supported and funded by the Universidad de Antioquia, Colombia [CODI-PRG13-2-08, 2013] and Fondo Nacional de Regalías de la República de Colombia [Ruta N 139C, 2014].

*María Bernarda Salazar and Mauricio Hernández V. are the Bioinstrumentation and Clinical Engineering Research Group - GIBIC, Bioengineering Department, Engineering Faculty, Universidad de Antioquia activates the fight-or-flight response, increases heart rate (HR) and sweating, dilates bronchi and pupils, and its fibers reach almost all organs and systems of the body [4]. On the contrary, the PNS is responsible for the body's resting state, internal organ regulation, and digestion. This system decreases HR, contracts bronchi and pupils, and helps maintain an equilibrium state of the body [4].

Organophosphate (OP) poisoning acts directly on the ANS and its action mechanism involves the inhibition of acetylcholinesterase enzymes (AChE), which breaks down acetylcholine (ACh) at cholinergic synapses [5]. The ACh is a neurotransmitter released by the terminal nerve endings of all postganglionic parasympathetic nerves and in both sympathetic and parasympathetic ganglia [5]. Severe OP poisoning is typically characterized by bronchospasms, hypotension or hypertension, salivation, pinpoint pupils, agitation, confusion, bradycardia or tachycardia, excessive sweating, respiratory failure, and coma [6]. Respiratory failure can be attributed to dysfunction of neuromuscular junctions, which is associated with muscle weakness and muscle paralysis [5] (The action mechanism of the OP is given in the Appendix). It is estimated that one third of all cases of OP poisoning requires mechanical ventilation [7]. To withdrawal ventilatory support (weaning), it is necessary to assess not only the respiratory muscle activity but also the cardiorespiratory condition of the patient during spontaneous breathing test (SBT).

The importance of measuring the heart rate variability is undeniable [8], [9], usually the HRV is performed to analyze drug administration effects [10] and as a diagnostic tool for diseases such as post-myocardial infarction [11], [12] and risk of sudden death [13]. There are reports using HRV as a predictor of successful SBT in non-intoxicated patients [14], [15], but they do not take into account quantitative information about respiratory muscle activity nor about the SNS or PNS action to evaluate the inability of the main muscle to support the load associated with spontaneous breathing.

There are mathematical models that characterize the cardiorespiratory mechanism incorporating the autonomic

UdeA; Calle 70 No. 52-21, Medellín, A.A. 1226, Colombia (correspondence email:bernarda.salazar@udea.edu.co).

Miguel Ángel Mañanas was with the Department of Automatic Control and the Biomedical Engineering Research Centre of the Universitat Politècnica de Catalunya, Calle Jordi Girona, 31, 08034, Barcelona, Spain.

Cesar Cortés Daza was with Hospital San Vicente Fundación, Calle 64 \# 51 D - 154 Medellín, Colombia. 
control system during rest, sleep, exercise or hypercapnia conditions [16], [17]. These are useful tools to understand the SNS and PNS engagement but they do not allow quantifying the magnitude of the action or effects of drugs or toxins on each one. Thus, in this article, we present an extended model of cardiovascular regulation for assessing the HRV during SBT in patients poisoned with $\mathrm{OP}$, considering quantification of the sympathetic - vagal engagement. The objective of our study was to model the HRV including the respiratory muscle activity through the sympathetic - vagal parameters in poisoned patients, using biopotentials of the diaphragm and sternocleidomastoid muscles. Understanding the sympathetic vagal balance and estimating the magnitude of its action would allow addressing pharmacological treatment through the patient's physiological response measured non-invasively.

\section{Populations AND ExPERIMENTAL Design}

\section{A. Patient group}

A database of twenty-one patients (17 men, 4 women; age $30.0 \pm 11.8$ years, weight $64.3 \pm 9.0 \mathrm{~kg}$, height $165.3 \pm 9.0 \mathrm{~cm}$ ) with organophosphorus poisoning was used. Patients were included in the study according to the following inclusion criteria: i) intoxication with OP, ii) undergoing mechanical ventilation, iii) absence of myopathy and thoracic trauma, iv) older than 16, and v) not pregnant.

This study was conducted under the approval of the Ethical Review Committee of the San Vicente Fundación Hospital and written informed consent was obtained from the patient's family for the publication of this study.

\section{B. Cardiac, respiratory and surface electromyographic signals}

Three-lead electrocardiographic (ECG) signals and electromyographic activity were recorded using a biopotential amplifier (Bagnoli EMG system, Delsys, Massachusetts, United States), coupled to an acquisition card (NI USB 6212) configured with a sampling frequency of $1024 \mathrm{~Hz}$. Surface electrodes were located in a bipolar configuration for each respiratory muscle of interest in the following positions: (a) right activity of the diaphragm was recorded between the seventh and eighth intercostal space, on the line located in the middle of the mid axillary line and the external clavicular line [18], [19]; (b) for the sternocleidomastoid muscle, electrodes were placed at $20 \%$ of the distance between the mastoid apophysis and the sternal notch and over the line that connects them [18].

The ventilatory signals (tidal volume, air flow and respiratory rate) were recorded with a HAMILTON-S1 ventilator (Hamilton Medical, Bonaduz, Switzerland).

\section{Study design}

The study consisted in measuring the HRV and respiratory muscle activity while assessing the patient's ability to breathe (SBT) receiving minimal ventilation support for $20 \mathrm{~min}$, with patients in supine position and the bed head elevated at least 35 degrees. The start of SBT was defined by clinical expertise based on ventilatory signal measurements used as classical predictors (e.g., rapid shallow breathing - RSB), but always assessing the absence of any of the following previous conditions: (i) respiratory rate lower than 8 breaths/min or higher than 35 breaths/min for 5 minutes or longer; (ii) hypoxemia (oxygen saturation measured by blood analysis lower than $88 \%$ for 5 minutes or longer); (iii) sudden changes in mental status; (iv) cardiac arrhythmia, and (v) two or more symptoms of respiratory distress such as bradycardia (lower than 60 beats/min), tachycardia (higher than 130 beats/min), paradoxical breathing, diaphoresis or dyspnea.

\section{METHOD}

\section{A. Structured Model}

This study was founded on the model proposed by Saul et al. in 1991 [17]. The structured of the original model (Model I) is schematically shown in Fig. 1. The Model I studies HR regulation and systemic blood pressure through a feedback control system that includes the action of arterial baroreceptors. The breathing is represented by volume or airflow which generates fluctuations in the arterial blood pressure (ABP), this vital process is attributed to changes in pressure and volume of the chest.

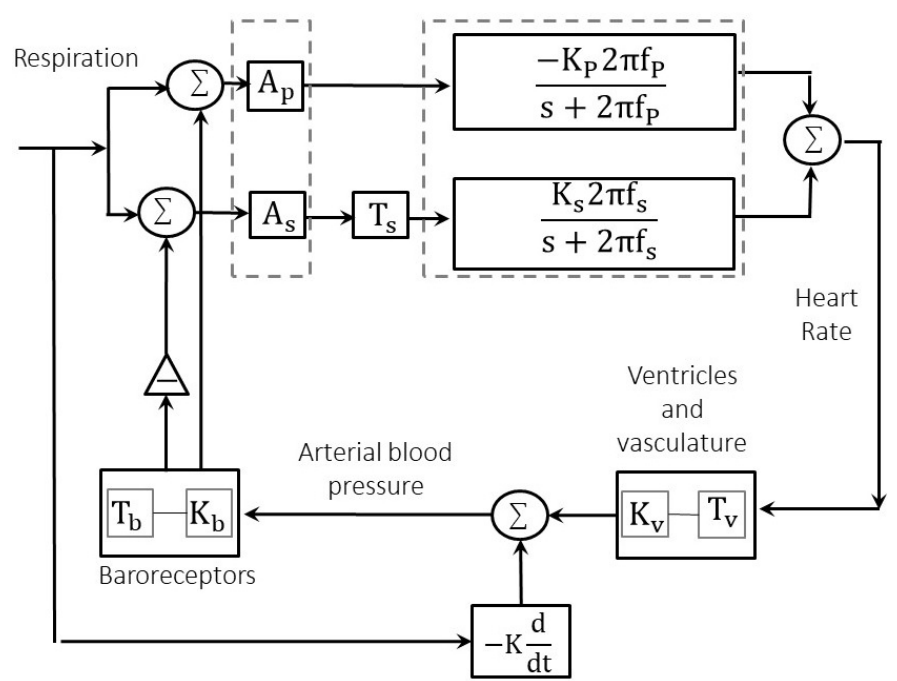

Fig. 1. (Model I) Model of closed-loop cardiovascular regulation published by Saul et al. [17]. Constant $K_{b}$ and delay $T_{b}$ of the Baroreptors; Constant $K_{v}$ and delay $T_{v}$ of the ventricles and vasculature; The parasympathetic and sympathetic system is a first-order low-pass filters with corner frequencies $\left(f_{p}, f_{s}\right)$ and constant gains $\left(\mathrm{A}_{\mathrm{p}}, K_{p}, K_{s}, A_{s}\right)$ and a delay $T_{s}$ to mimic latency in the sympathetic response.

The mechanical effects of respiration are modeled as a respiration derivative [17]. The properties of the arterial vasculature and heart ventricles are modeled as a constant $K_{v}$ in series with a fixed delay $T_{v}$ [17], which represents the influence of the pulse duration on stroke volume [20]. The baroreflex control (constant $K_{b}$ and delay $T_{b}$ ) acts directly on the HR and is linked both to the parasympathetic and the sympathetic control as a simple summation with weight factors, $A_{p}$ and $A_{s}$, respectively. Saul et al. characterized the contribution of these systems in healthy subjects under pharmacological blocked conditions and changes in posture. They modeled the parasympathetic and sympathetic system as first-order low-pass filters with corner frequencies $\left(f_{p}, f_{s}\right)$ and constant gains $\left(K_{p}\right.$, 
$K_{s}$ ) [17], in which the sum effects increase HR during inspiration and decrease HR during expiration in a healthy subject [20], recreating the dynamics of the sinoatrial (SA) node. The authors consider a delay $T_{s}$ to mimic latency in the sympathetic response.

In this study, we propose to modify the order and structure of the parasympathetic and sympathetic transfer functions and the weight factors of baroreflex control (see Fig. 2), since according to experimental results, a first-order system was not enough to model the frequency characteristics of the heart rate in poisoned patients.

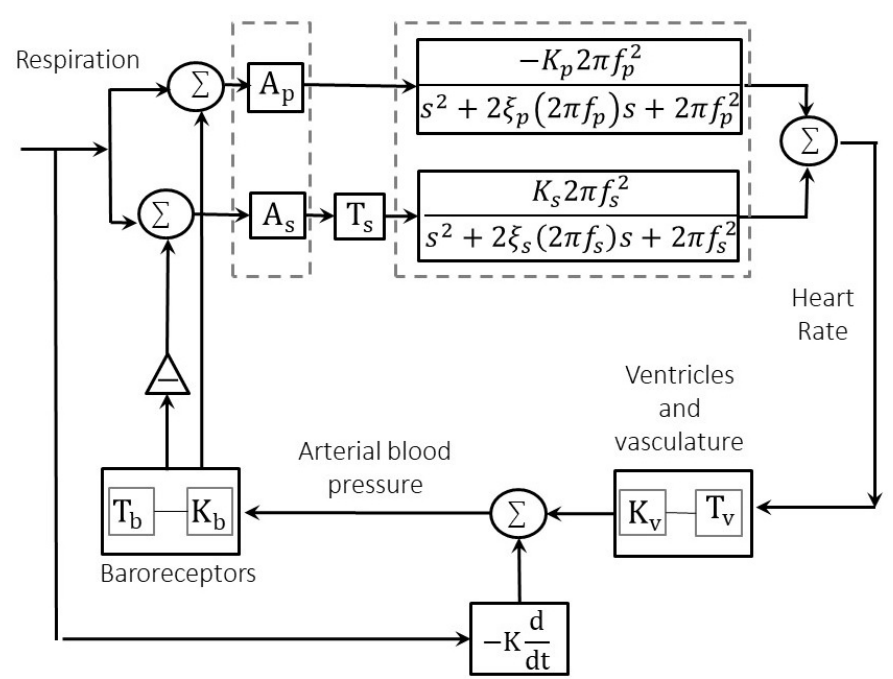

Fig. 2. (Model II) Model of closed-loop cardiovascular regulation proposing that respiration induces heart rate fluctuations. This scheme considers the model published by Saul et al. [17] and the changes proposed in this article are enclosed by dashed boxes. The parasympathetic and sympathetic system is a second-order low-pass filters with corner frequencies $\left(f_{p}, f_{s}\right)$, constant gains $\left(\mathrm{A}_{\mathrm{p}}\right.$, $K_{p}, K_{s}, A_{s}$ ), a delay $T_{s}$ to mimic latency in the sympathetic response and the damping constants $\xi_{p}$ and $\xi_{s}$.

\section{B. Identification process}

Breathing induces heart rate fluctuations, although the effect in poisoned patients is unknown. The model fitted for healthy subjects by Saul et al. [17] requires changes in structure and parameter values to be fitted to this population according to a preliminary test. The identification process was carried out in the frequency domain to characterize the effect in the HRV using the cost function represented in (1). The optimization process involved the model parameters adjustment to experimental data (see Fig. 3) and the identification of the system from a set of candidate models [21], [22] (transfers functions with different order, from 1 to 5) for the parasympathetic and the sympathetic system.

The cost function measures differences between simulated (SIM) and experimental (EXP) data in frequency, as follows:

$$
\begin{aligned}
& J=\sum_{k=0}^{p-1}\left(\frac{\left|H_{E X P}\left(w_{k}\right)\right|-\left|H_{S I M}\left(w_{k}\right)\right|}{\left|H_{E X P}\left(w_{k}\right)\right|}\right)^{2}+ \\
& \left(\frac{\varphi\left(H_{E X P}\left(w_{k}\right)\right)-\varphi\left(H_{S I M}\left(w_{k}\right)\right)}{\varphi\left(H_{E X P}\left(w_{k}\right)\right)}\right)^{2}
\end{aligned}
$$

Where $\left|H\left(w_{k}\right)\right|$ and $\varphi\left(w_{k}\right)$ represent magnitude and phase, respectively, and $\mathrm{k}$ is the sample number.
Being $H\left(w_{k}\right)$ the transfer function in the frequency domain, obtained using the following equation:

$$
H\left(w_{k}\right)=\frac{S_{u y}\left(w_{k}\right)}{S_{u u}\left(w_{k}\right)}
$$

Where $S_{u y}$ is the cross-spectrum between input $(u)$ - output (y) and $S_{u u}$ is the power spectrum of the input (auto-spectrum).

The optimization was performed by minimizing $J$ in function of eight parameters (for order 2 structure), which define the parasympathetic and sympathetic response in the Model II (see Fig. 2).

$$
J=J\left(K_{p}, A_{p}, f_{p}, \xi_{p}, K_{s}, A_{s}, f_{s}, \xi_{s}\right)
$$

The implementation of this controller was carried out by Sequential Quadratic Programing (SQP) [23]-[25], using Matlab. This technique allowed minimizing the scalar function $J$ within a region specified by constraints and bounds. In order to guarantee results with physiological sense, the searching space for $\left\{K_{p}, A_{p}, f_{p}, \xi_{p}, K_{s}, A_{s}, f_{s}, \xi_{s}\right\}$ was defined within the range reported by other authors [17], [20]. The damping constants was optimized between:

$$
\left\langle 0<\xi_{p}<1\right\rangle,\left\langle 0<\xi_{s}<1\right\rangle
$$

Being SQP a deterministic algorithm, it could be potentially sensitive to the seeds; these were randomly generated with uniform distributions. When a premature convergence to a local minimum was presented, an automatic random re-initialization was applied to improve the reached solution, if it was possible [23]. Finally, the optimization process was performed 40 times in each case (see Fig. 3) to guarantee the optimization reproducibility and that parameter values fall within the physiological range.

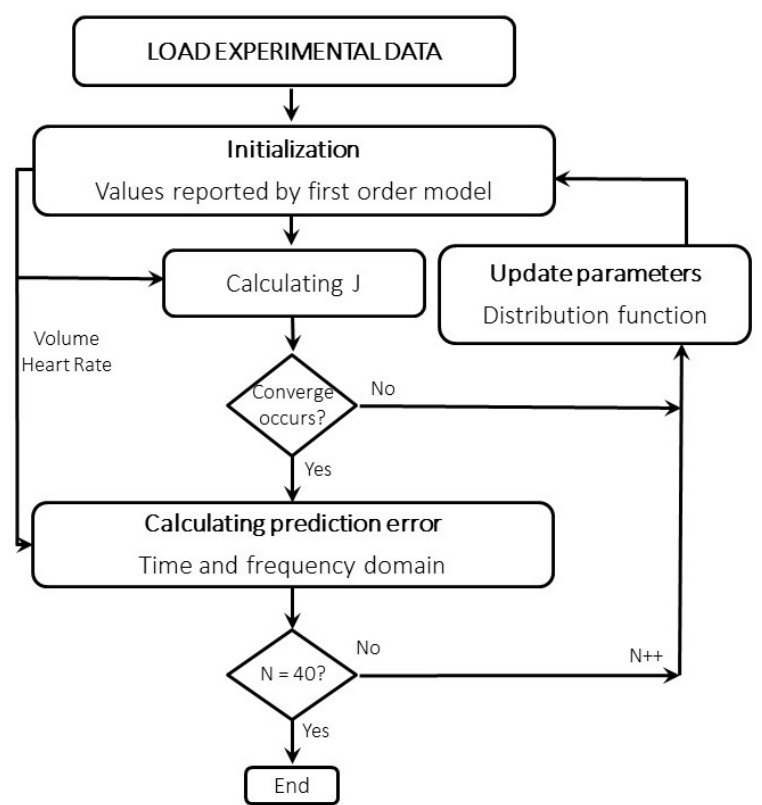

Fig. 3. Flowchart of the optimization process implemented to identify the parameter values of the second order heart rate variability model.

\section{Validation}

With the aim of statistically comparing the fitness of the 
model and the mathematical relationship between the parameters and sEMG variables, the Wilcoxon Mann Whitney nonparametric test was applied with a significant level of $\alpha=$ 0.05 .

To guarantee the reproducibility of the results, the parameters without a clear relationship with sEMG variables were set in median of random value of the probability distribution function (obtained in the optimization phase), this process was carried out 40 times per-patient.

\section{INDEXES}

\section{A. Heart Rate Variability}

The spectral power density (PSD) of electrocardiographic signals was estimated using the Burg's method [26], this technique models the time series using an autoregressive process, where the PSD is estimated from the model [27]. Most of the signal power is from 0 to $0.4 \mathrm{~Hz}$. Three main spectral components are distinguished in a spectrum calculated from short term recordings (2 to 5 minutes): Very Low Frequency (VLF), Low Frequency (LF) and High Frequency (HF) components, the frequency ranges are shown in Table I.

TABLE I

PARAMETERS IN THE FREQUENCY DOMAIN [32].

\begin{tabular}{llc}
\hline \hline \multicolumn{1}{c}{ Variable } & \multicolumn{1}{c}{ Description } & $\begin{array}{c}\text { Frequency } \\
\text { Range }(\mathrm{Hz}))\end{array}$ \\
\hline Total power $\left(\mathrm{ms}^{2}\right)$ & $\begin{array}{l}\text { Power density in all } \\
\text { frequency range }\end{array}$ & $0.04-0.4$ \\
VLF power $\left(\mathrm{ms}^{2}\right)$ & Total power density & $\leq 0.04$ \\
LF power $\left(\mathrm{ms}^{2}\right)$ & $\begin{array}{l}\text { Total power density } \\
\text { LF power / (Total }\end{array}$ & $0.04-0.15$ \\
LF power & power - VLF power $) \times$ & $0.04-0.15$ \\
normalized $(\%)$ & $\begin{array}{l}\text { Peak Frequency } \\
\text { LF peak }(\mathrm{Hz})\end{array}$ & $0.04-0.15$ \\
HF power $\left.(\mathrm{ms})^{2}\right)$ & Total power density & $0.15-0.4$ \\
HF power & $\begin{array}{l}\text { HF power / (Total } \\
\text { power - VLF power }) \times\end{array}$ & $0.15-0.4$ \\
normalized $(\%)$ & 100 & $0.15-0.4$ \\
HF peak $(\mathrm{Hz})$ & $\begin{array}{l}\text { Peak Frequency } \\
\text { Ratio between LF and } \\
\text { HF band powers }\end{array}$ & - \\
\hline \hline
\end{tabular}

\section{B. Muscular variables}

The root mean square (RMS) value is used as a measurement associated with the signal's amplitude [28]. In this study, the ratio between inspiratory and expiratory RMS values $\left(\mathrm{RMS}_{\mathrm{i} / \mathrm{e}}\right)$ is an indicator of the muscle fiber activation. $\mathrm{RMS}_{\mathrm{i} / \mathrm{e}}$ achieved values higher than one when the muscle was active asumming that the muscle relaxed during expiration. The ratio was calculated according to the following expression:

$$
R M S_{i / e}=\frac{\sqrt{\frac{1}{N} \sum_{i=1}^{N}\left|s E M G_{i}(i)\right|^{2}}}{\sqrt{\frac{1}{N} \sum_{i=1}^{N}\left|s E M G_{e}(i)\right|^{2}}}
$$

Where $\mathrm{N}$ is the sample number in the half-cycle.

The engagement between respiratory muscles was evaluated using the squared Pearson correlation coefficient between the rectified sEMG (Env) of the diaphragm (Dia) and sternocleidomastoid (Strn) muscles [29]:

$$
r_{\text {Dia, Strn }}^{2}=\left(\frac{C\left(E n v_{D i a}, E n v_{S t r n}\right)}{\sqrt{C\left(E n v_{D i a}, E n v_{D i a}\right) * C\left(E n v_{S t r n}, E n v_{S t r n}\right)}}\right)^{2}
$$

Where $\mathrm{C}$ is the covariance matrix.

This coefficient, $r_{\text {Dia, Strn }}^{2}$, weighs the synchronism importance between implied respiratory muscles during ventilation. When contractions are synchronous, it means that both main and accessory respiratory muscles work together to support spontaneous breathing.

In all cases, the patients drank an unknown amount of organophosphorus pesticide during a suicidal attempt. On admission, gastric lavage was performed and activated charcoal was given promptly. During the spontaneous breathing test, patients had the heart rate in a range of $65-120$ beats per minute, the respiratory frequency between $10-24$ breaths per minute and the tidal volume $\left(\mathrm{V}_{\mathrm{T}}\right)$ between $0.32-0.8$ liters. These ranges represent a high dispersion of the cardiac and ventilatory response in the study population. The $\mathrm{V}_{\mathrm{T}}$ shows the random nature of the spontaneous breathing (see Fig. 4), its frequency content reflects that the highest energy is in the HF band and occurs in the mean respiratory rate. The power spectral density of HR has an inverse distribution compared to the respiratory signal distribution: greater peak in the LF than in the HF band.
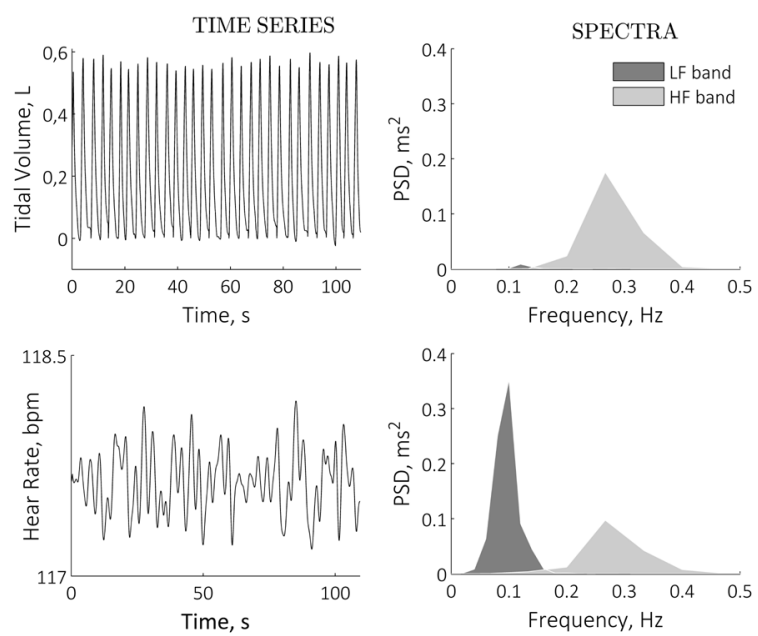

Fig. 4. Representative time series and spectra of tidal volume (Liters, L) and heart rate (Beats per minute, bpm). Most of the signals power is in a range of 0 to $0.5 \mathrm{~Hz}$. The main spectral components are Low Frequency (LF band, 0.04 $-0.15 \mathrm{~Hz}$ ) and High Frequency (HF band, $0.15-0.4 \mathrm{~Hz}$ ) components. PSD: power spectral density.

Those parameters with low variance among patients were $A_{s}$, $\xi_{p}$, and $\xi_{s}$, which mean and standard deviation values were 26 $\pm 1.3,0.9 \pm 0.08$ and $0.89 \pm 0.10$, respectively. Its deviations are very low compared to the mean value, i.e. their optimal value is similar for most patients. Although the searching space was extended, this result remained, so that it could be inferred that the mean value for these parameters are a global minimum. 
The relationship between optimal parameters and electromyographic activity in patients poisoned with OP was explored both in the temporal and frequency domain. The $r_{D i a, S t r n}^{2}$ represents the grade of linear correlation between Dia and Strn on the weaning test, where a low correlation coefficient suggests poor engagement between Dia and Strn. The ratio between inspiratory and expiratory RMS values, $\mathrm{RMS}_{\mathrm{i} / \mathrm{e}}$ (Dia), suggests that the muscle contracts during inspiration when $\mathrm{RMS}_{\mathrm{i} / \mathrm{e}}$ is greater than 1 .

The Fig. 5 shows the strength of the relationship among muscle activity and model parameters as follows: (i) $r_{D i a, S t r m}^{2}$ $f_{p}$, (ii) $\mathrm{RMS}_{\mathrm{i} / \mathrm{e}}(\mathrm{Dia})-\mathrm{A}_{\mathrm{p}}$ and (iii) $\mathrm{RMS}_{\mathrm{i} / \mathrm{e}}(\mathrm{Dia})-f_{s}$. Fig. 5.A shows that when the diaphragm contractions are stronger, according to increases of $\mathrm{RMS}_{\mathrm{i} / \mathrm{e}}$, the sympathetic frequency increases. An opposite behavior is shown in Fig. 5.B, showing that the $A_{p}$ decreases when $\mathrm{RMS}_{\mathrm{i} / \mathrm{e}}$ (Dia) increases; and the parasympathetic frequency, $f_{p}$, decreases when the synchronism between respiratory muscles, $r_{D i a, \text { Strm }}^{2}$, increases (see Fig. 5.C). The last result suggests that when the patient needs to use the main muscle and the accessory respiratory muscle simultaneously to support the demand for spontaneous breathing, the parasympathetic system decreases its action.
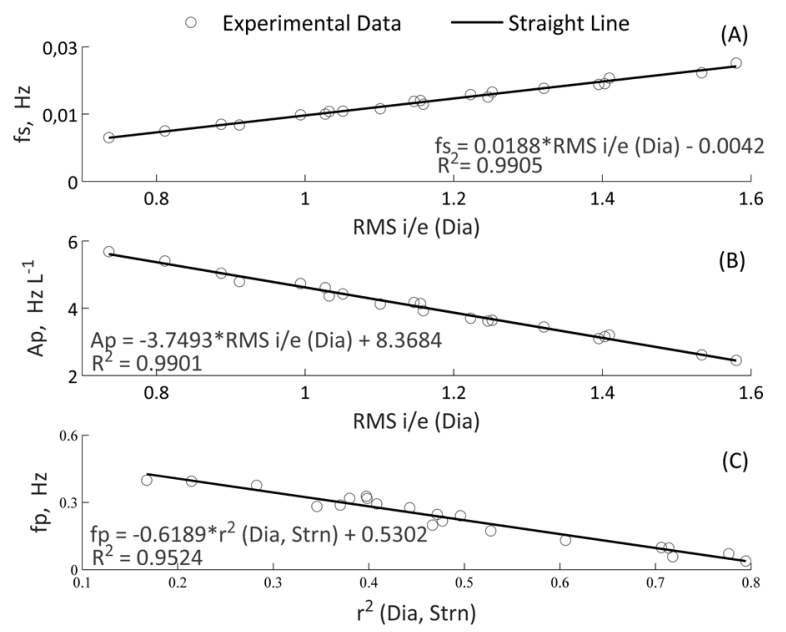

Fig. 5. Relationship between optimal parameter (Model II) and electromyographic variables. The squared Pearson correlation coefficient $\left(\mathrm{R}^{2}\right)$ between the rectified amplitudes of sEMG for two different muscles: diaphragm (Dia) and sternocleidomastoid (Strn) muscles; $\mathrm{RMS}_{\mathrm{i} / \mathrm{e}}(\mathrm{Dia})$ : the ratio between inspiratory and expiratory RMS values; fp: parasympathetic corner frequency; Ap: parasympathetic weight factors of baroreflex control; and fs: sympathetic corner frequency.

From obtained optimal values in all studied samples, parasympathetic transfer function gain, $\mathrm{K}_{\mathrm{p}}$, fits a Kernel distribution (Fig. 6.A), this produces a nonparametric probability density estimate [30] that adapts itself to the data with Kernel normal and bandwidth equal to 1.5336 . Sympathetic transfer function gain, $\mathrm{K}_{\mathrm{s}}$, has a normal distribution with a mean and standard deviation of 20.21 and 10.62, respectively (Fig. 6.B).
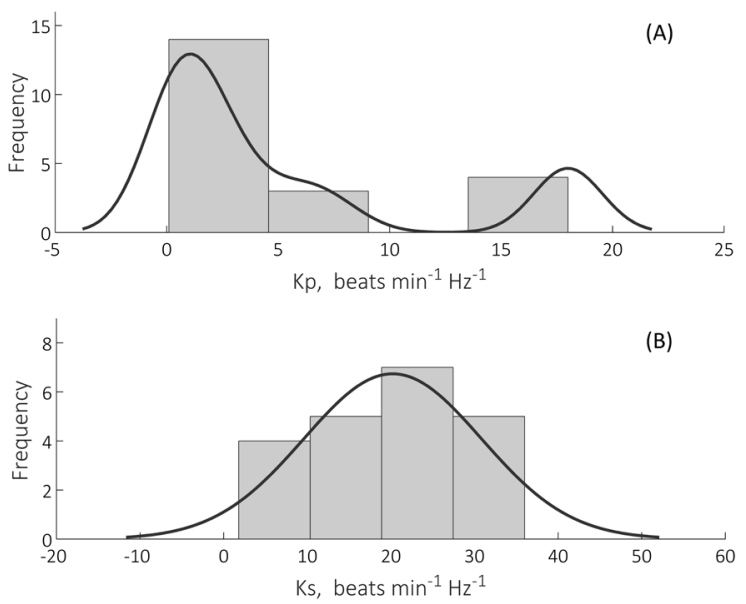

Fig. 6. Probability distribution function for the following optimal parameters of the Model II: (A) Kernel distribution for Kp (parasympathetic constant gain), Kernel normal and bandwidth: 1.5336; (B) Normal distribution for Ks (sympathetic constant gain), mean: 20.21 and standard deviation 10.62 .

The frequency response shows differences between the sympathetic and parasympathetic system both in magnitude and phase during spontaneous breathing. The sympathetic response displays a magnitude decrement and a phase delay increment as frequency increases, this behavior is more notable above 0.15 $\mathrm{Hz}$. The parasympathetic heart rate control is characterized by a constant magnitude phase delay at frequency from 0 to 0.5 $\mathrm{Hz}$.

As a visual reference, the experimental data of one patient is compared to HR predictions by simulations models (I and II) in Fig. 7. The HR amplitude and frequency variations predicted by the model using the tidal volume and electromyographic information (Dia and Strn) mimic the characteristics of the experimental data. There are small discrepancies in the LF band, mainly in the total energy contained in this frequency, although, the energy distribution in comparison to the real signal is preserved between bands.

The above suggests that the changes proposed to the original model simulates the vagal - sympathetic balance in relation to cardiac variability during spontaneous breathing in intoxicated patients. This corresponds with the magnitude and phase response of the transfer function (in average) because the response in the LF band is product of the interaction between the parasympathetic and sympathetic system.

Table II shows the frequency variables calculated from the experimental and simulated HR (Model I and II); these variables are usually evaluated make a vagal - sympathetic balance diagnosis in HR short-term 5-min recordings. Only the spectrum's peaks from LF and HF bands may be used as a measure about autonomic modulation of $\mathrm{HRV}$ in shorter recordings. Its maximum and minimum values are presented in each variable as well as the prediction error with respect to variables measured for experimental data.

According to prediction error results, the Model II shows a good fit to the population, the total power and its distribution in terms of low and high frequency bands has an error lower than $20 \%$, compared to Model I which has errors greater than $200 \%$. For Model II, the most significant error occurs in the HF band, 
which is reflected in the prediction error associated to the $\mathrm{LF} / \mathrm{HF}$ ratio, perhaps because the frequency response of the parasympathetic system does not present significant changes in magnitude and phase when the respiratory rate rises or falls during spontaneous breathing test.
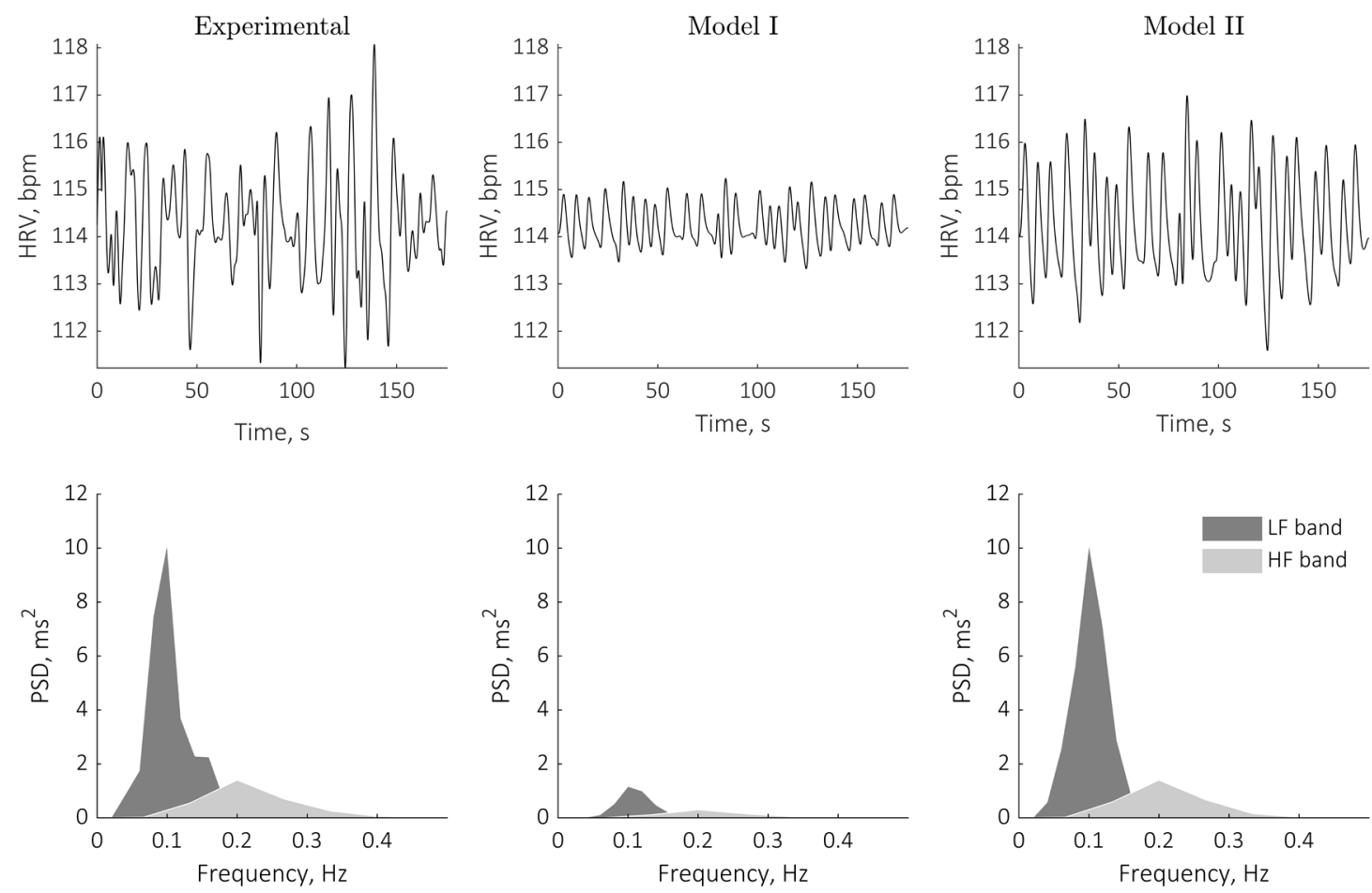

Fig. 7. Comparison of experimental data and simulation results (Model I and II) of representative time series and spectra of the heart rate (Beats per minute, bpm) of a random patient. The main spectral components are Low Frequency (LF band, dark gray, $0.04-0.15 \mathrm{~Hz}$ ) and High Frequency (HF band, light gray, $0.15-0.4 \mathrm{~Hz})$.

TABLE II

PREDICTION ERROR IN FREQUENCY DOMAIN CALCULATED IN THE ANALYSIS OF SHORT-TERM RECORDINGS (5 MIN) OF THE HEART RATE SIMULATED WITH THE ORIGINAL AND PROPOSED MODELS (I AND II RESPECTIVELY). LOW FREQUENCY (LF BAND, $0.04-0.15$ Hz) AND HIGH FREQUENCY (HF BAND, 0.15 - 0.4 HZ).

\begin{tabular}{|c|c|c|c|c|c|}
\hline \multirow{2}{*}{ Variable } & \multirow{2}{*}{$\begin{array}{c}\text { Experimental } \\
{[\min , \max ]}\end{array}$} & \multicolumn{2}{|c|}{ Model I } & \multicolumn{2}{|c|}{ Model II } \\
\hline & & $\begin{array}{l}\text { Simulated } \\
{[\min , \max ]}\end{array}$ & $\begin{array}{c}\text { Error } \\
(\%)\end{array}$ & $\begin{array}{l}\text { Simulated } \\
{[\min , \max ]}\end{array}$ & $\begin{array}{c}\text { Error } \\
(\%)\end{array}$ \\
\hline LF peak $(\mathrm{Hz})$ & {$[0.058,0.124]$} & {$[0.060,0.140]$} & {$[3.448,12.90]$} & {$[0.060,0.124]$} & {$[0.000,3.448]$} \\
\hline LF power normalized (\%) & {$[65.98,97.90]$} & {$[9.834,93.38]$} & {$[4.616,85.09]$} & {$[72.46,97.75]$} & {$[0.230,9.830]$} \\
\hline HF peak $(\mathrm{Hz})$ & {$[0.200,0.333]$} & {$[0.200,0.400]$} & {$[0.000,20.12]$} & {$[0.200,0.400]$} & {$[0.000,20.00]$} \\
\hline HF power normalized $(\%)$ & {$[2.028,34.02]$} & {$[6.612,90.16]$} & {$[164.9,226.0]$} & {$[2.250,27.53]$} & {$[10.94,19.06]$} \\
\hline $\mathrm{LF} / \mathrm{HF}$ ratio & {$[1.939,48.31]$} & {$[0.109,14.12]$} & {$[70.77,94.37]$} & {$[2.632,43.45]$} & {$[10.06,35.70]$} \\
\hline
\end{tabular}

\section{DISCUSSION}

The aim of this study was to model the HRV considering the relationship between heart rate dynamics and respiratory muscle activity through the sympathetic - vagal parameters in poisoned patients. Many studies have shown that power spectrum analysis of the heart rate variability provides important information about autonomic nervous system activity [31], [32]. The starting point of this work was the model proposed by Saul et al. in 1991, this model is focused on characterizing the respiratory sinus arrhythmia (RSA) in healthy subjects [17]. The relationship between RSA and HRV can be measured as a variation of the HF band in HR record
[33]. Therefore, with the purpose of modeling the HRV characteristics both in low and high frequency bands, a modification in the sympathetic and parasympathetic transfer functions has been proposed and validated using experimental data. Two parameters were added to the model, $\xi_{p}$ and $\xi_{s}$, corresponding to the damping factor in a second-order system.

We propose that oscillations, whether part of in LF or HF band can be actively compensated for by vagal-sympathetic control. This approach has been referred by Piovesan et al. in a third-order model to represent the biomechanical properties of muscles and tendons [34]. The $\xi_{p}$ and $\xi_{s}$ may be related with future studies with vasodilation and vasoconstriction produced by sympathovagal regulation, an important characteristic that 
Saul et al., do not included in the original model and could play an important role in poisoned or under pharmacological treatment patients.

The optimal parameters of the first-order low-pass filter [17] for the parasympathetic and the sympathetic systems were obtained under different conditions for this study (Model I): healthy subjects with pharmacological autonomic blockage and under posture changes. However, we consider these as physiological values but we extended the searching scope in the SQP algorithm to analyze if the optimal parameters found after minimization would correspond to a local or a global minimum. Serna et al. [23] found that the SQP algorithm was more efficient in comparison to other algorithms in the prediction of pattern changes within a respiratory cycle.

The definition of the model parameters - Model II considering the patient's condition is relevant because the HRV is influenced not only by factors such as age or gender, but also by the physiological state and drug pharmacodynamics [31]. Taking into account the relationship between relaxation and contraction in muscles that depends on vagal and sympathetic action, respectively, the physiological state of the poisoned patient can be evaluated through the respiratory muscle activity measurement [35]. The muscle activity in the diaphragm and sternocleidomastoid muscles were recorded with surface electromyography according to previous studies that evaluated the activity of muscles involved in breathing during SBT in mechanically ventilated patients [36]-[38]. Two variables related to muscular activity during SBT in patients poisoned with organophosphorus compounds have been presented in this paper: the $r_{D i a, \text { str }}^{2}$ coefficient and the classical $\mathrm{RMS}_{\mathrm{i} / \mathrm{e}}$ ratio. In previous studies theses variables have been used as an indicator of respiratory muscles activity in a case report [39] and as a good measure of the sEMG signal amplitude both in 12 mechanically ventilated patients [40] and in a population of 23 patients poisoned with organophosphate [38]. These findings show that the $\mathrm{RMS}_{\mathrm{i} / \mathrm{e}}$ (Dia) and $r_{\text {Dia, Srrn }}^{2}$ have a strong relationship with parasympathetic and sympathetic parameters, which complements their usefulness in critical patient monitoring. Particularly, the results showed two possible response patterns, the first one characterized by the presence of diaphragm's weak contractions $\left(\mathrm{RMS}_{\mathrm{i} / \mathrm{e}} \approx 1\right)$ and low engagement between the diaphragm and sternocleidomastoid muscle $\left(r_{\text {Dia, Strn }}^{2}<1\right)$. These suggest a state of muscle relaxation that drives the inability of the main muscle to support the load associated with spontaneous breathing. According to the results (Model II), patients with these characteristics have the highest values of frequency $\left(f_{p}\right)$ and gain $\left(\mathrm{A}_{\mathrm{p}}\right)$ of the parasympathetic branch. This pattern agrees with what is known about the parasympathetic action: regulates rest functions and it is predominant during a quiet state [4]. On the contrary, the sympathetic action had a greater relationship with an increase in the diaphragm's activation: $f_{s}$ increases as $\mathrm{RMS}_{\mathrm{i} / \mathrm{e}}$. Although the spontaneous breathing test does not imply physical activity such as riding a bicycle, this puts the patient in a stress situation because during the gradual withdrawal of ventilatory support, he assumes his breath.

It is possible that the patient presents signs of increased work of breathing or distress, therefore requiring the use of the accessory muscles. These responses are usually activated by the sympathetic system, which prepares the body for the stress response [4].

During spontaneous breathing test, the patients had an upper torso inclination of $35^{\circ}-40^{\circ}$. Results published by Kubota et al. suggested that slight flexion of the upper torso in Fowler's position activates respiratory function and increases the contribution of vagal nerve activity to the cardiovascular system [41]. This could explain the low variability of sympathetic weight factors of baroreflex control $\left(A_{s}\right)$.

The results obtained from the HRV simulation with the Model II, including the respiratory muscle activity of the diaphragm and sternocleidomastoid muscles are comparable with the experimental data, specifically in the frequency variables evaluated in the HRV analysis. The increase in time between heartbeats implies HR decreases with an accompanying increase of HRV. The opposite occurs when the HR increases [32]. The values for the frequency variables reflects that the $\mathrm{HF}$ power corresponds with respiratory activity related to the frequency characteristics of heart rate. Mccraty et al. [32] suggests that lower HF power is associated with stress, panic, anxiety, or worry. This is consistent with our results, because the HF band has the lower power normalized in all patients. It is known that the SBT causes many distressing symptoms, such as: pain, agitation, lack of sleep, and especially anxiety [42].

The sympathetic system decreases its influence in rhythms above $0.15 \mathrm{~Hz}$, while the parasympathetic system is affected between 0.04 to $0.4 \mathrm{~Hz}$. Some authors have suggested that the LF band reflects sympathetic activity [43], although most investigators believe it reflects sympathovagal activity [44], [45]. Even though the patients during SBT were not under the pharmacological autonomic blocked, 16/21 patients received atropine therapy $(0.5-27 \mathrm{mg})$ on arrival in the intensive care and the effects of atropine may not have disappeared completely even six weeks after exposure [46]. Whereby the LF power could be related with sympathetic action, taking into account the effects the atropine and effort associated with the spontaneous breathing test.

\section{CONCLUSION}

To find a quantitative relationship between respiratory muscle activity and the cardiorespiratory response during the recovery period of poisoning, through the quantification of sympathovagal participation in the cardiac response variability, its use could give light in improving the efficiency of therapeutic treatments, more specifically in the ventilatory strategy. The extended model of cardiovascular regulation presented introduces an alternative approach to assess the sympathetic - parasympathetic balance through the heart rate variability complemented with information about the respiratory muscle engagement. The diaphragm and sternocleidomastoid activity measured by surface 
electromyography can reflect and quantify the sympathetic vagal engagement during spontaneous breathing test. This model may be used to continuously assess (non-subjective) the muscle weakness caused by organophosphates poisoning and the clinical state of the sympathetic and parasympathetic system (considering the pharmacological treatment) possibly achieving a shorter mechanical ventilation time, establishing requirements for mechanical support adapted to the daily needs of each patient.

\section{APPENDIX}

In the following link, the GIBIC group shares a 3D animation of the action mechanism of the Organophosphorus compounds produced during the developing of this thesis: https://youtu.be/8R9fNhvkXog.

\section{REFERENCES}

[1] A. Camm et al., "Heart rate variability: standards of measurement, physiological interpretation and clinical use. Task Force of the European Society of Cardiology and the North American Society of Pacing and Electrophysiology," Circulation, vol. 93, no. 5, pp. 1043-1065, 1996.

[2] G. Berntson et al., "Heart rate variability: Origins, methods and interpretive caveats.," Psychophysiology, vol. 34, pp. 623-648, 1997.

[3] T. Laitio, J. Jalonen, T. Kuusela, and H. Scheinin, "The role of heart rate variability in risk stratification for adverse postoperative cardiac events," Anesth. Analg., vol. 105, no. 6, pp. 1548-1560, 2007.

[4] L. K. McCorry, "Physiology of the Autonomic Nervous System," Am. J. Pharm. Educ., vol. 71, no. 4, pp. 1-11, 2007.

[5] E. J. Hulse, J. O. J. Davies, a. J. Simpson, A. M. Sciuto, and M. Eddleston, "Respiratory Complications of Organophosphorus Nerve Agent and Insecticide Poisoning. Implications for Respiratory and Critical Care," Am. J. Respir. Crit. Care Med., vol. 190, no. 12, pp. 1342-1354, 2014.

[6] M. Eddleston, N. a Buckley, P. Eyer, A. H. Dawson, J. V. Peter, and G. John, "Management of acute organophosphorus pesticide poisoning.," Lancet, vol. 371, no. 9612, pp. 597-607, Feb. 2008.

[7] U. a. D. D. Munidasa, I. B. Gawarammana, S. a. M. Kularatne, P. V. R. Kumarasiri, and C. D. a. Goonasekera, "Survival Pattern in Patients with Acute Organophosphate Poisoning Receiving Intensive Care," Clin. Toxicol., vol. 42, no. 4, pp. 343-347, Jan. 2004.

[8] M. P. Tarvainen, J. P. Niskanen, J. A. Lipponen, P. O. Ranta-aho, and P. A. Karjalainen, "Kubios HRV - Heart rate variability analysis software," Comput. Methods Programs Biomed., vol. 113, no. 1, pp. 210-220, 2014.

[9] S. Y. Sim et al., "Estimation of Circadian Body Temperature Rhythm Based on Heart Rate in Healthy, Ambulatory Subjects," IEEE J. Biomed. Heal. Informatics, vol. 21, no. 2, pp. 407-415, Mar. 2017.

[10] D. Almoznino-Sarafian et al., "Magnesium administration may improve heart rate variability in patients with heart failure," Nutr. Metab. Cardiovasc. Dis., vol. 19, no. 9, pp. 641-645, 2009.

[11] N. L. Oliveira, F. Ribeiro, A. J. Alves, M. Teixeira, F. Miranda, and J. Oliveira, "Heart rate variability in myocardial infarction patients: Effects of exercise training," Port. J. Cardiol., vol. 32, no. 9, pp. 687-700, 2013.

[12] Szi-Wen Chen and Shih-Chieh Chao, "Compressed Sensing Technology-Based Spectral Estimation of Heart Rate Variability Using the Integral Pulse Frequency Modulation Model," IEEE J. Biomed. Heal. Informatics, vol. 18, no. 3, pp. 1081-1090, May 2014.

[13] F. Lombardi, T. H. Makikallio, R. J. Myerburg, and H. V Huikuri, "Sudden cardiac death: role of heart rate variability to identify patients at risk," Cardiovasc. Res., vol. 50, no. 2, pp. 210-217, 2001.

[14] C. T. Huang, Y. J. Tsai, J. W. Lin, S. Y. Ruan, H. D. $\mathrm{Wu}$, and C. J. Yu, "Application of heart rate variability in patients undergoing weaning from mechanical ventilation," Crit Care, vol. 18, no. 1, p. R21, 2014.

[15] T. A. Lennie and M. Hardin-pierce, "Heart rate variability as a predictor od cardiac dysrhythmias during weaning from mechanical ventilation," Pulmonaty Crit. Care, vol. 24, no. 2, pp. 118-128, 2015.

[16] L. Cheng, O. Ivanova, H.-H. Fan, and M. C. K. Khoo, "An integrative model of respiratory and cardiovascular control in sleep-disordered breathing.," Respir. Physiol. Neurobiol., vol. 174, no. 1-2, pp. 4 28, Nov. 2010.

[17] J. P. Saul, R. D. Berger, P. Albrecht, S. P. Stein, M. H. Chen, and R. J. Cohen, "Transfer function analysis of the circulation: unique insights into cardiovascular regulation.," Am. J. Physiol., vol. 261, no. 4 Pt 2, pp. H1231-H1245, 1991.

[18] J. F. Alonso, M. A. Mañanas, D. Hoyer, Z. L. Topor, E. $\mathrm{N}$. Bruce, and others, "Evaluation of respiratory muscles activity by means of cross mutual information function at different levels of ventilatory effort.," IEEE Trans. Biomed. Eng., vol. 54, no. 9, pp. 1573-82, Sep. 2007.

[19] P. W. Hodges and S. C. Gandevia, "Pitfalls of intramuscular electromyographic recordings from the human costal diaphragm," Clin. Neurophysiol., vol. 111, no. 8, pp. 1420-4, Aug. 2000.

[20] P. Chalacheva and M. C. K. Khoo, "An extended model of blood pressure variability: incorporating the respiratory modulation of vascular resistance.," Conf. Proc. ... Annu. Int. Conf. IEEE Eng. Med. Biol. Soc. IEEE Eng. Med. Biol. Soc. Annu. Conf., vol. 2013, pp. 3825-8, 2013.

[21] Z. Philips et al., "Review of guidelines for good practice in decision-analytic modelling in health technology assessment.," Health Technol. Assess., vol. 8, no. 36, p. iii-iv, ix-xi, 1-158, 2004.

[22] J. J. DiStefano and E. M. Landaw, "Multiexponential, and noncompartmental modeling. I . Methodological limitations and physiological interpretations," Am. $J$. Physiol., vol. 84, pp. 651-664, 2010. 
[23] L. Serna et al., "Novel Modeling of Work of Breathing for Its Optimization During Increased Respiratory Efforts," IEEE Syst. J., no. 99, pp. 1-11, 2014.

[24] P. E. Gill, W. Murray, M. A. Saunders, and M. H. Wright, "Procedures for optimization problems with a mixture of bounds and general linear constraints," $A C M$ Trans. Math. Softw., vol. 10, no. 3, pp. 282-298, 1984.

[25] L. Y. Serna, M. Á. Mañanas, J. Marín, A. M. Hernández, and S. Benito, "Optimization techniques in respiratory control system models," Appl. Soft Comput., vol. 48, 2016.

[26] R. Merletti, D. Farina, and C. Disselhorst-Klug, "Single-Channel Techniques for Information Extraction from the Surface EMG Signal," in Electromyography: Physiology, Engineering, and NonInvasive Applications, M. Parker and R. Merletti, Eds. New Jersey: IEEE PRESS, 2004, pp. 169-204.

[27] J. G. Proakis and D. G. Manolakis, "Filtros Adaptativos," in Tratamiento Digital de Señales, 4a ed., Madrid, España: Pearson Pretince Hall, 2007, pp. 785-850.

[28] C. J. De Luca, "The use of surface electromyography in biomechanics," J. Appl. Biomech., vol. 13, no. July 1993, pp. 1-38, 1997.

[29] V. K. Ingle and J. G. Proakis, Digital Signal Processing Using MATLAB, vol. 4. 2000.

[30] X. Xu, Z. Yan, and S. Xu, "Estimating wind speed probability distribution by diffusion-based kernel density method," Electr. Power Syst. Res., vol. 121, pp. 28-37, 2015.

[31] J. Kranjec, S. Beguš, G. Geršak, and J. Drnovšek, "Non-contact heart rate and heart rate variability measurements: A review," Biomed. Signal Process. Control, vol. 13, no. 1, pp. 102-112, 2014.

[32] R. Mccraty and F. Shaffer, "Heart Rate Variability: New Perspectives on Physiological Mechanisms, Assessment of Self-regulatory Capacity, and Health Risk," Glob. Adv. Heal. Med., vol. 4, no. 1, pp. 46-61, 2015.

[33] M. Moldovan, S. Spulber, V. Saravan, R. Iosifescu, A. M. Zluagrean, and L. Zluagrean, "The relationship between respiratory sinus arrhythmia and heart rate during anesthesia in rat.," Rom. J. Physiol. Physiol. Sci., vol. 41, no. 1-2, pp. 31-39, 2004.

[34] D. Piovesan, A. Pierobon, and F. A. Mussa Ivaldi, "Critical Damping Conditions for Third Order Muscle Models: Implications for Force Control," J. Biomech. Eng., vol. 135, no. 10, p. 101010, 2013.

[35] C. Collet, F. Di Rienzo, N. El Hoyek, and A. Guillot, "Autonomic nervous system correlates in movement observation and motor imagery," Front. Hum. Neurosci., vol. 7, no. July, pp. 1-17, 2013.

[36] G. Bellani et al., "Estimation of patient's inspiratory effort from the electrical activity of the diaphragm.," Crit. Care Med., vol. 41, no. 6, pp. 1483-91, 2013.

[37] M. Dres, M. Schmidt, A. Ferre, J. Mayaux, T. Similowski, and A. Demoule, "Diaphragm electromyographic activity as a predictor of weaning failure," Intensive Care Med., vol. 38, no. 12, pp. 20172025, 2012.

[38] M. B. Salazar Sánchez, A. M. Hernández Valdivieso, and M. Á. Mañanas Villanueva, "Assessment of mechanically ventilated patients intoxicated with organophosphates by a novel surface electromyographic index," J. Crit. Care, vol. 41, pp. 260-267, 2017.

[39] M. B. Salazar Sánchez, A. M. Hernández Valdivieso, M. Á. Mañanas Villanueva, and A. F. Zuluaga Salazar, "Potential clinical application of surface electromyography as indicator of neuromuscular recovery during weaning tests after organophosphate poisoning," Rev. Bras. Ter. Intensiva, vol. 29, no. 2, pp. 253-258, 2017.

[40] M. Schmidt et al., "Dyspnea and surface inspiratory electromyograms in mechanically ventilated patients," Intensive Care Med., vol. 39, no. 8, pp. 1368-1376, 2013.

[41] S. Kubota, Y. Endo, and M. Kubota, "Effect of upper torso inclination in Fowler's position on autonomic cardiovascular regulation," J. Physiol. Sci., vol. 63, no. 5, pp. 369-376, 2013.

[42] B. Hetland, R. Lindquist, and L. Chlan, "The influence of music during mechanical ventilation and weaning from mechanical ventilation: A review.," Hear. Lung, vol. 44, no. 5, pp. 416-425, 2015.

[43] D. L. Eckberg, "Sympathovagal Balance," Circulation, vol. 96, pp. 3224-3232, 1997.

[44] M. L. Appel, R. D. Berger, J. P. Saul, J. M. Smith, and R. J. Cohen, "Beat to beat variability in cardiovascular variables: Noise or music?," J. Am. Coll. Cardiol., vol. 14, no. 5, pp. 1139-1148, 1989.

[45] S. Akselrod, D. Gordon, F. A. Ubel, D. C. Shannon, A. C. Berger, and R. J. Cohen, "Power Spectrum Analysis of Heart Rate Fluctuation: A Quantitative," Science, vol. 213, no. July, pp. 220-222, 1981.

[46] S. S. Jayasinghe and K. D. Pathirana, "Autonomic function following acute organophosphorus poisoning: a cohort study.," PLoS One, vol. 7, no. 5, p. e37987, Jan. 2012. 